\title{
ON THE SIGNIFICANCE OF PSEUDOFEMALE BEHAVIOR IN THE NEOTROPICAL COCKROACH GENERA BLABERUS, ARCHIMANDRITA AND BYRSOTRIA
}

\author{
By Peter W. Wendelken ${ }^{1}$ and Robert H. Barth, Jr. ${ }^{2}$
}

The basic constituents of successful courtship behavior in most species of cockroaches studied thus far are as follows: (1) The male contacts the female. (2) The male courts the female and gives a "full wing raising display," elevating his wings to a nearly vertical position and turning away from the female. (3) The female shows the "mounting and feeding" response. She straddles the male's exposed abdomen and moves forward. As she mounts, the female's mouthparts and palps are closely applied to the male's abdominal tergites and are worked vigorously. (In some species, the female feeds upon secretions of specialized tergal glands.) (4) The male backs beneath the mounting and feeding female, extending his abdomen backward in copulatory thrusts. (5) Genital connection is achieved and the pair execute a turning operation to assume the opposed copulatory position (male and female facing in opposite directions). The opposed position persists throughout copulation which may last for an hour or more (Barth 1968a, Wendelken 1976, Simon and Barth 1977a). Females and males are generally suspected of producing sex pheromones of varying degrees of volatility which function in the release of certain aspects of courtship behavior. The relative contributions of chemical and tactile stimuli to the release of the various stages in the courtship sequence vary considerably among different species (Barth 1968a). As a rule, female sex pheromones are important in release of male courtship behavior, including the full wing raising display (Barth 1968a, 1970; Wendelken 1976; Simon and Barth 1977b). And male sex pheromones are believed to be involved in release of the female mounting and feeding response which serves to position the female such that the male may achieve genital connection (Roth and Dateo 1966, Barth 1968a). The tactile stimuli produced by the female's mounting and feeding response play an

'P.O. Box 26, Marietta, OH 45750

${ }^{2}$ Department of Zoology, University of Texas, Austin, TX 78712

Manuscript received by the editor June 20, 1985 
important role in the release of the male's backing movements and copulatory thrusts (Barth 1964, 1968a; Wendelken 1976; Simon and Barth 1977a).

In cockroaches, the term "homosexual behavior" has been used in reference to the behavior of males directing male courtship patterns toward other males (Barth 1964). "Pseudofemale behavior" has been used to designate the appearance in the male of motor patterns which are characteristic of female sexual behavior-i.e., the "mounting and feeding" response. Pseudofemale mounting and feeding release backing and copulatory thrusts by the displaying male. However, genital connection is only rarely achieved and in such cases lasts only a few seconds (Barth 1964). Homosexual and pseudofemale behavior are of widespread occurrence in cockroaches. Homosexual behavior may be at least partially explicable in terms of female sex pheromone becoming attached to the cuticle of males exposed to females. The female sex pheromone would tend to release courtship behavior in males coming into antennal contact with these pheromone-tinged males. Thus, to some extent, homosexual behavior may be an artifact of the courtship situation (Roth and Willis 1952, Simon and Barth 1977b).

No satisfactory explanation has been advanced to account for the perplexing prevalence of pseudofemale behavior in cockroaches. The elementary form of male mounting and feeding behavior, as described above, has been reported for the following species: Periplaneta americana (L.) (Roth and Willis 1952, Barth 1970, Simon and Barth 1977a); Periplaneta brunnea Burmeister and Periplaneta fuliginosa (Serville) (Barth 1970, Simon and Barth 1977a); Periplaneta japonica and Periplaneta australasiae (F.) (Simon and Barth 1977a); Blatta orientalis L. (Roth and Willis 1952, Barth 1970, Simon and Barth 1977a); Supella longipalpa (F.) (Roth 1952); Latiblattella angustifrons Hebard (Willis 1970); Eurycotis floridana (Walker) (Barth 1968b); Blattella germanica (L.) (Roth and Willis 1952); Parcoblatta fulvescens (Saussure and Zehntner) (Wendelken and Barth 1971); Byrsotria fumigata (Guérin) (Barth 1964); Leucophaea maderae (F.) and Nauphoeta cinerea (Olivier) (Roth and Barth 1967). This list is not necessarily all inclusive but it does indicate the widespread occurrence of the elementary form of pseudofemale behavior in cockroaches. In none of these instances was pseudofemale behavior described as being a component of the male repertoire of aggressive behavior. 
This paper discusses the significance of a previously unreported form of pseudofemale behavior in the neotropical cockroach genera Blaberus, Archimandrita, and Byrsotria. These genera belong to the Tribe Blaberini (Blaberidae, Blaberinae) according to Roth (1970a).

\section{Materials AND Methods}

Stock cultures were maintained as described by Barth (1964) for Byrsotria fumigata. Observations of behavior were made during the dark phase of a 12 hour day/night cycle under red illumination in specially designed observation chambers constructed of transparent lucite plastic $(39.5 \times 26.8 \times 14.5 \mathrm{~cm}$ deep $)$ with a removable center partition dividing the chamber into two halves allowing segregation of the sexes prior to an observation period (for details, see Barth 1964).

\section{Courtship in Tribe Blaberini}

Very elaborate and complex preliminary male courtship behavior is found in Blaberus discoidalis Serville, B. parabolicus Walker, B. giganteus (L.), B. craniifer Burmeister, Archimandrita tessellata Rehn, and Byrsotria fumigata. In addition, in these species there is frequently vigorous aggressive behavior between males at the site of courtship of a female. These phenomena are considered to be a consequence of sexual selection acting on the males. "Sexual selection" here refers to a selective advantage for characters producing a competitively unequal advantage in both epigamic and intrasexual interactions associated with mating behavior. In spite of the complexities of male behavior in these species, the terminal events leading to copulation (the copulation sequence) follow a basic cockroach pattern (Wendelken 1976). Preliminary male courtship behaviors are released by a female sex pheromone (Barth 1964, 1970; Wendelken 1976). If a courted female is receptive, she signals receptivity by moving or turning toward the courting male and simultaneously antennating him. This receptive female behavior releases the male's culminating courtship display: the "full wing raising display." In the full wing raising display, the male pivots 180 degrees to face away from the female and simultaneously raises his wings to a vertical position. The receptive female, in probable response to a male sex pheromone, then proceeds to "mount and feed" in a forward direction over the exposed dorsal surface of the male's abdo- 
men (Wendelken 1976). She straddles the male's abdomen as she mounts and makes "feeding" movements with her mouthparts on the male's exposed abdominal tergites. (Unlike certain cockroach species the males do not possess macroscopically visible tergal glands in these species.) As she mounts the male backs beneath her. When her feeding activities reach the region of the first tergite, the male makes rhythmic protrusions of his hooked right phallomere. Genital connection is achieved and the pair performs a turning operation which results in the opposed copulatory position (Wendelken 1976).

It should be emphasized that the sequence of events initiated by a female's display of receptive behavior toward a courting male will ordinarily proceed quickly to copulation in the absence of disruption by other animals. In the family Blaberidae, the ovoviviparous reproductive cycle is distinguished by oviposition of the ootheca into a brood sac where incubation proceeds until hatching occurs about one to three months later. A female which copulates loses the capacity for sexual receptivity until after parturition.

\section{Results}

In these species, aggression between males in courtship situations is very intense and often violent. However, there is little in the way of aggressive interactions between males and females. Among themselves, males engage in fighting, chasing, and fleeing. Pseudofemale behavior was found to play a conspicuous role in the overall context of male-male aggression as outlined below.

Courting males frequently interfere with the courtship of rival males. Interference may take place at any stage of courtship. The more disruptive forms of intervention are enumerated as follows: (1) Frequently, a male will attack another male which is directing preliminary courtship to a female. The attacker jumps on top of his opponent, grasping his tegmina and/or pronotum and biting him. (2) A male may attack another male which is performing a full wing raising display by showing "aggressive pseudofemale behavior." Before the female has a chance to respond, the aggressive male mounts and feeds upon the exposed abdominal tergites of the displaying male as would a receptive female. However, unlike a receptive female, when his head reaches the region of the first abdominal tergite, he bites the elevated wings or the region of the first tergite of 
his opponent. This causes the displaying male to run rapidly forward in an attempted escape. (3) Another form of assault occurs in which a male attacks a second male which is performing a full wing raising display. Before the female has an opportunity to respond, the attacking male leaps onto the displaying male's abdomen (this does not resemble the elementary pattern of pseudofemale mounting and feeding), attempting to bite him (particularly his raised wings). Such an action causes the displaying male to run off rapidly. (4) A male may disrupt a copulation sequence in progress by attacking as a female mounts a displaying male. The attacker may break up the sequence by climbing onto the displaying male's abdomen from the side, pushing the female off, and then biting his wings, causing him to run off. Or a male may directly attack the mounting female. (5) Attacks may be directed against copulating pairs. Typically, the attacker jumps upon the copulating animals. Sometimes these attacks are focused at the juncture between the male and female. In two cases in B. craniifer, such attacks caused separation of a pair which had just assumed the opposed position. Apparently their genitalia were not as yet securely attached. Attacks on copulating pairs commencing after the initial few seconds following assumption of the opposed position were never observed to cause separation of a copulating pair. The observed forms of male intervention in the courtship of other males are shown for each species in Table 1.

In tests of interspecific sexual interactions in which females were presented with a choice of conspecific and heterospecific males, interspecific pseudofemale behavior was observed in three combinations. In one cross, a male $B$. giganteus mounted a displaying $B$. discoidalis male and bit his wings. Other forms of interspecific malemale aggression were observed between these two species.

In another cross, $B$. giganteus males showed pseudofemale mountings of $B$. craniifer males and vice versa. In at least one case, a mounting $B$. craniifer male launched an attack directed against the wings of a displaying $B$. giganteus male. There was also reciprocal interspecific disruption of copulation sequences by males of these two species.

In an intergeneric combination, Byrsotria fumigata males were observed mounting Blaberus discoidalis males. It was not observed whether these pseudofemale episodes were aggressive in nature. 


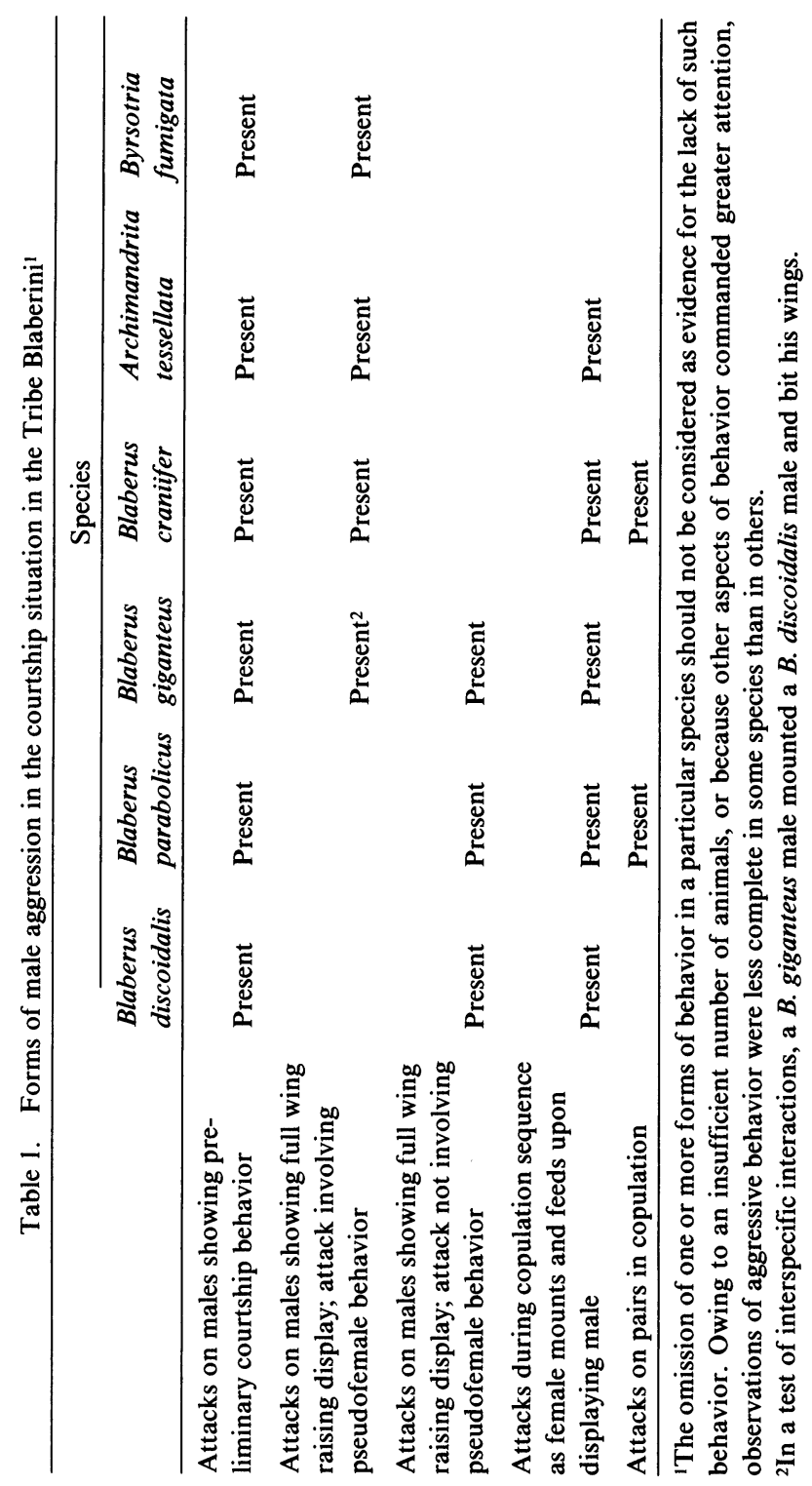




\section{Discussion}

The conditions under which observations were made in the laboratory appear to approximate rather well important conditions found in some natural populations. Gautier (1974) studied a high density population of Blaberus colosseus in a Trinidad cave and found that the cockroaches "live in groups including males, females at different phases of their reproductive cycle and older nymphs. These males are not territorial, only very few are observed away from the group." Sexually receptive females were found to travel as much as several meters in a day and this caused modifications in the distribution of males. In cockroaches (e.g., Blaberus spp.) with a volatile female sex pheromone, the female sex pheromone acts to attract males but is effective over a distance of a few feet at most (Barth 1968a). In the high density population studied by Gautier (1974), clearly a number of males would come into contact with a sexually receptive female. Gautier (1978) studied another high density cave population of $B$. colosseus in Trinidad. He found that "males are not territorial on sites" which they occupy and that the interindividual relationships of males are of short duration and low intensity. And, significantly, "the presence of a female changes male/male relationships quickly and profoundly by stimulating aggressiveness" in the males. Gautier (1978) also observed a number of cases in which two males were simultaneously in contact with a single female. He concluded that his field observations confirmed his finding in the laboratory that the presence of a female releases aggressive behavior between males.

Sexual selection may lead to the evolution of intense male-male aggression. This appears to have been the case in the genera Blaberus, Archimandrita, and Byrsotria (Wendelken 1976). In the species reported on here, direct confrontations take place between competing males in the presence of a female. Males aggressively interfere with the courtship of other males, including foiling the female mounting and feeding sequence. It is in this context that we find pseudofemale mounting and feeding with subsequent biting of the displaying male. Aggressive pseudofemale behavior appears to function as one of an array of methods by which a male can discourage the copulation of a receptive female with one of his rivals and thus increase his own chances of copulation. If a male mounts a displaying rival, the female is precluded from doing so. In other words, one 
way for a male to prevent a receptive female from mounting and feeding upon a displaying rival (and thus copulating) is for the male to do the mounting and feeding himself. When a receptive female responds to a rival's full wing raising display, it is too late for a male to effectively court the female. At this point the only way to preserve the opportunity to direct effective courtship to the female is to first disrupt the ongoing copulation sequence involving the rival male. In a small population of cockroaches of these species, only one or several receptive females will be present at any given time. A male performing pseudofemale behavior prevents an imminent loss of a scarce resource, i.e., the receptive female. The receptive female consequently remains available to respond to subsequent courtship by the pseudofemale performer.

A second aspect of aggressive pseudofemale behavior is related to the fact that a courting male is quite vulnerable to attack when giving a full wing raising display. A male employing pseudofemale mounting and feeding behavior is able to "lure" the displaying male into a still more vulnerable position and then unleash a sudden attack at the very moment the displaying rival is attempting copulation. The result is that the displaying male is driven away and prevented, at least for the moment, from copulating. Such an encounter might also exert a negative influence upon the propensity for subsequent courtship by the victim of the pseudofemale attack. To sum up, the pseudofemale actor engages in behavior that simultaneously increases his fitness and decreases that of his competitor.

A male may engage in pseudofemale behavior as a prelude to an attack or he may attack directly. There appears to be a gradation of responses between these two alternative behavior patterns. Which is chosen may depend upon (a) the level of aggressive tendencies in the attacking male, (b) the temporal proximity to the onset of female mounting, and (c) the relative position of the two males.

In the species reported on here, female sex pheromones are interspecifically effective in releasing male courtship behavior (Barth 1970, Wendelken 1976). Interspecific differences in male sex pheromones play a vital role in reproductive isolation among these species. With the exception of the combination $B$. parabolicus $\times B$. discoidalis, females either refuse or are reluctant to mount and feed in response to the displays of heterospecific males (Wendelken 1976). These findings are generally consistent with the results of interspe- 
cific mating tests performed by Roth (1970b). That pseudofemale behavior is shown interspecifically suggests that this response is not released by male sex pheromone or that males respond to a wider spectrum of male sex pheromones than do females. In the event that members of two species come into contact, it may be adaptive for a male to be able to disrupt heterospecific courtship if this increases the probability that a conspecific female will be available for copulation with that male.

Thornhill (1979) reports an example of pseudofemale behavior in scorpionflies which functions to allow the pseudofemale performer to steal nuptial prey from rival males and then use the prey for copulation. There is a parallel to the present work in that the objective of prey theft can be accomplished by several means including but not limited to pseudofemale behavior.

Pseudofemale behavior in Blaberus, Archimandrita, and Byrsotria is an example of a seemingly abnormal behavior turning out on closer inspection to be adaptive. It may be that pseudofemale behavior (whether or not accompanied by an actual attack) serves similar ends in other cockroach species. Future workers should keep in mind that pseudofemale behavior need not lead to an outright attack in order to contribute to the copulatory success of the performing male. In species with an overall lower intensity of malemale aggression, pseudofemale behavior might be exhibited without the overt aggression so prominent in the Tribe Blaberini. Simply occupying a displaying male's abdomen prevents the female from copulating with the rival and buys time for the pseudofemale performer.

\section{ACKNOWLEDGEMENTS}

The research reported in this paper is part of a dissertation submitted by $\mathrm{P}$. W. W. for the Ph. D. degree. Thanks are expressed to J. L. Larimer, D. D. Thiessen, and T. H. Hamilton for reading an earlier version of the manuscript. The sources of the animals from which the stock cultures were derived are as follows: B. discoidalisF. A. McKittrick, Cornell University; B. parabolicus - L. M. Roth, collected in Brazil on Phase C of the Alpha Helix Expedition to the Amazon in 1967; B. giganteus-Harvard University culture originally from L. M. Roth; collection by R. H. Barth and F. A. McKittrick in Panama in 1962; culture from O. P. Breland, The University 
of Texas at Austin; B. craniifer-Harvard University culture originally from L. M. Roth; collection by D. Simon in Yucatan, Mexico in 1968; A. tessellata-collection by R. H. Barth and F. A. McKittrick in Panama in 1962; B. fumigata-Harvard University culture originally from L. M. Roth. R. H. B. and F. A. McK. thank STRI for the use of their facilities in Panama.

\section{SUMMARY}

This paper presents an account of a previously unreported form of pseudofemale behavior in cockroaches and comments on its possible adaptive significance. Females in most species of cockroaches must play an active role in order for copulation to occur. A receptive female "mounts and feeds" upon the exposed dorsal abdominal surface of a male performing the "full wing raising display". This serves to position the female so that the male may achieve genital connection. The appearance in males of the female pattern of mounting and feeding is known as "pseudofemale" behavior. Remarkably intense male-male aggression in courtship situations is found in the neotropical genera Blaberus, Archimandrita, and Byrsotria of the Tribe Blaberini (Blattaria, Blaberidae, Blaberinae). This can be explained as the result of sexual selection. Courting males frequently disrupt the courtship of rival males. Males may attack at any stage of the courtship of their rivals. In this context, pseudofemale behavior appears to function as part of an array of aggressive tactics males may employ to prevent the scarce receptive females from copulating with their rivals and thus increase their own prospects for achieving copulation. In these genera, pseudofemale behavior operates as follows. First, a male mounting a displaying rival effectively monopolizes that male's abdomen and precludes the receptive female from mounting. Second, after the pseudofemale mounting and feeding sequence has placed the displaying male in a highly vulnerable position, the pseudofemale performer, unlike a receptive female, attacks the displaying male by biting his wings and/or the region of the first abdominal tergite. This causes the displaying male to flee.

\section{REFERENCES Cited}

BARTH, R. H., JR. 1964. The mating behavior of Byrsotria fumigata (Guérin) (Blattidae, Blaberinae). Behaviour 23: 1-30. 
BARTH, R. H., JR. 1968a. The comparative physiology of reproductive processes in cockroaches. Part I. Mating behavior and its endocrine control. Advances in Reproductive Physiol. 3: 167-207.

BARTH, R. H., JR. 1968b. The mating behavior of Eurycotis floridana (Walker) (Blattaria, Blattoidea, Blattidae, Polyzosteriinae). Psyche 75: 274-84.

BARTH, R. H., JR. 1970. The mating behavior of Periplaneta americana (Linnaeus) and Blatta orientalis Linnaeus (Blattaria, Blattinae), with notes on the mating behavior of 3 additional species of Periplaneta and interspecific action of female sex pheromones. Z. Tierpsychol. 27: 722-48.

Gautier, J. V. 1974. Etude comparée de la distribution spatiale et temporelle des adultes de Blaberus atropos et B. colosseus (Dictyopteres) dans cinq grottes de l'ile de Trinidad. Rev. Comp. Animal 9: 237-58.

GaUtier, J. V. 1978. Le comportement social de Blaberus colosseus en milieu naturel; plasticité du système social. Insectes Sociaux 25: 289-301.

Rотн, L. M. 1952. The tergal gland of the male cockroach, Supella supellectilium. J. Morph. 91: 469-78.

Rотн, L. M. 1970a. The male genitalia of Blattaria. IV. Blaberidae: Blaberinae. Psyche 77: 308-42.

Roth, L. M. 1970b. Interspecific mating in Blattaria. Ann. Ent. Soc. Amer. 63: 1282-1285.

Roth, L. M., AND R. H. BARTH, JR. 1967. The sense organs employed by cockroaches in mating behavior. Behaviour 28: 58-94

Roth, L. M., AND G. P. DAteo. 1966. A sex pheromone produced by males of the cockroach, Nauphoeta cinerea. J. Ins. Physiol. 12: 255-65.

Roth, L. M., AND E. R. Willis. 1952. A study of cockroach mating behavior. Am. Midl. Nat. 47: 66-129.

Simon, D., AND R. H. BARTh. 1977a. Sexual behavior in the cockroach genera Periplaneta and Blatta. I. Descriptive aspects. Z. Tierpsychol. 44: 80-107.

Simon, D., AND R. H. BARTH. 1977b. Sexual behavior in the cockroach genera Periplaneta and Blatta. II. Sex pheromones and behavioral responses. Z. Tierpsychol. 44: 162-77.

ThORNhill, R. 1979. Adaptive female-mimicking behavior in a scorpionfly. Science 205: 412-4.

WendelKen, P. W. 1976. The evolution of courtship phenomena in Blaberus and related genera with reference to sexual selection. Ph.D. Thesis. University of Texas, Austin, TX. 257 p.

Wendelken, P. W., AND R. H. Barth. 1971. The mating behavior of Parcoblatta fulvescens (Saussure and Zehntner) (Blattaria, Blaberoidea, Blattellidae, Blattellinae). Psyche 78: 319-29.

WiLLIS, E. R. 1970. Mating behavior of three cockroaches (Latiblattella) from Honduras. Biotropica 2: 120-8. 

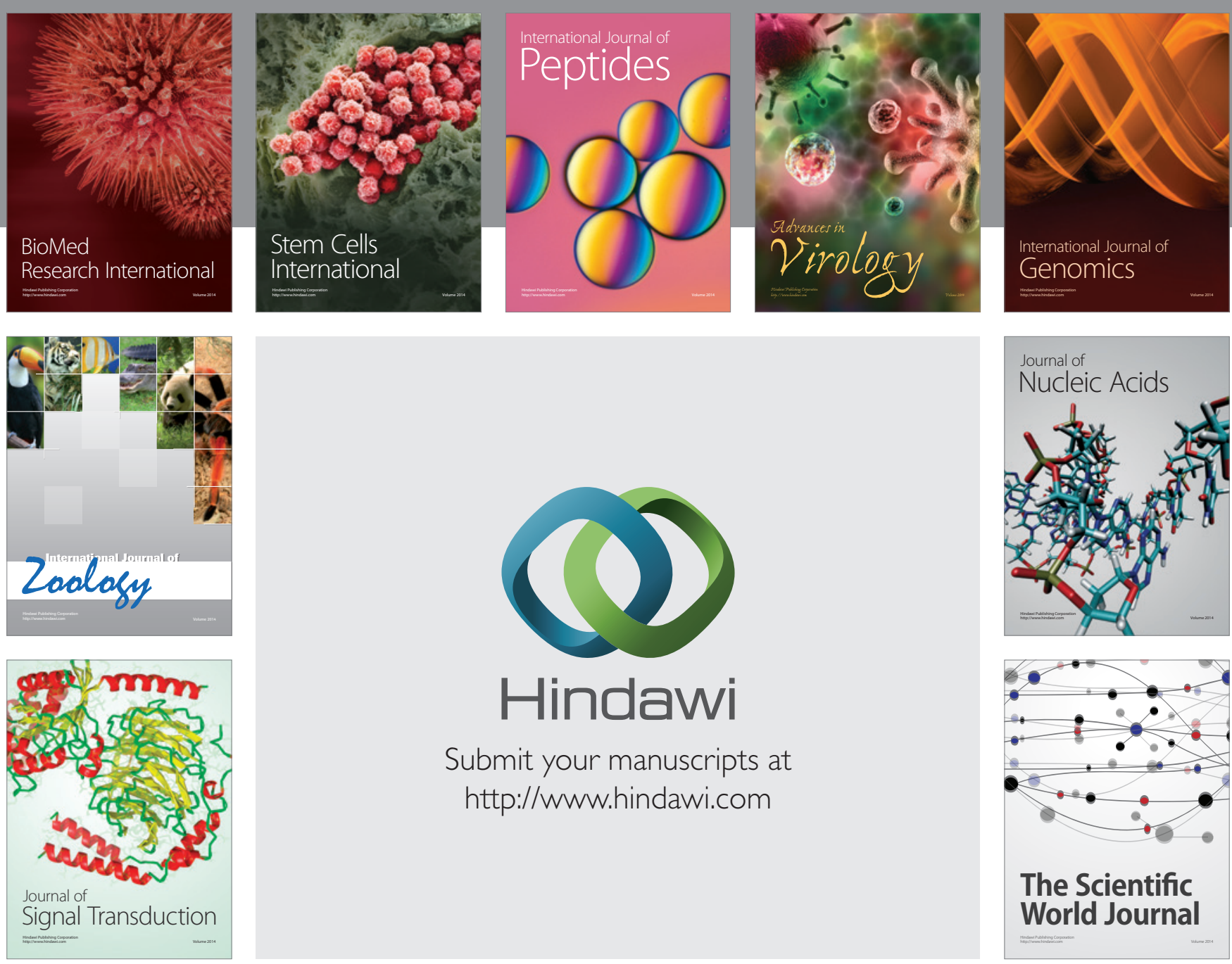

Submit your manuscripts at

http://www.hindawi.com
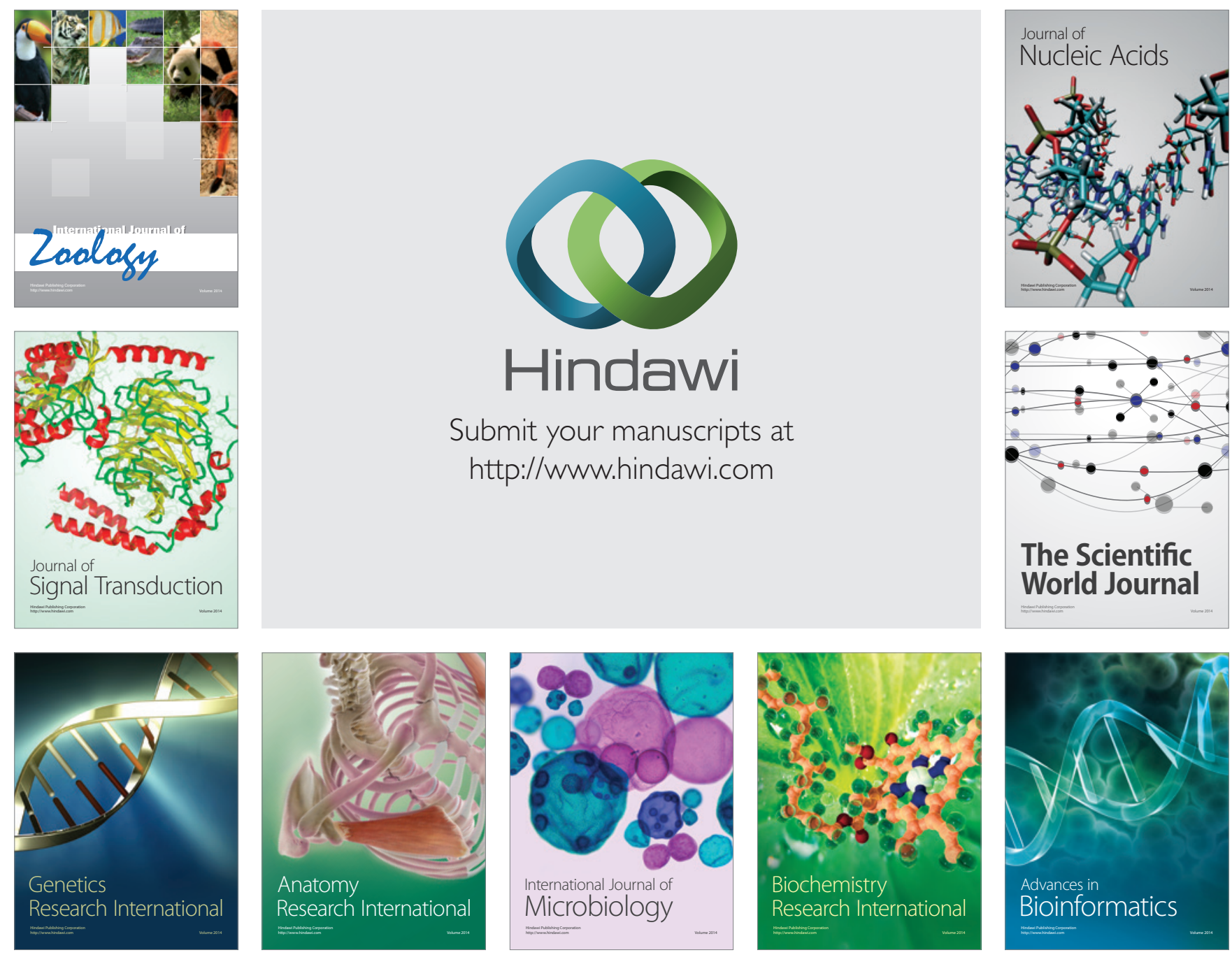

The Scientific World Journal
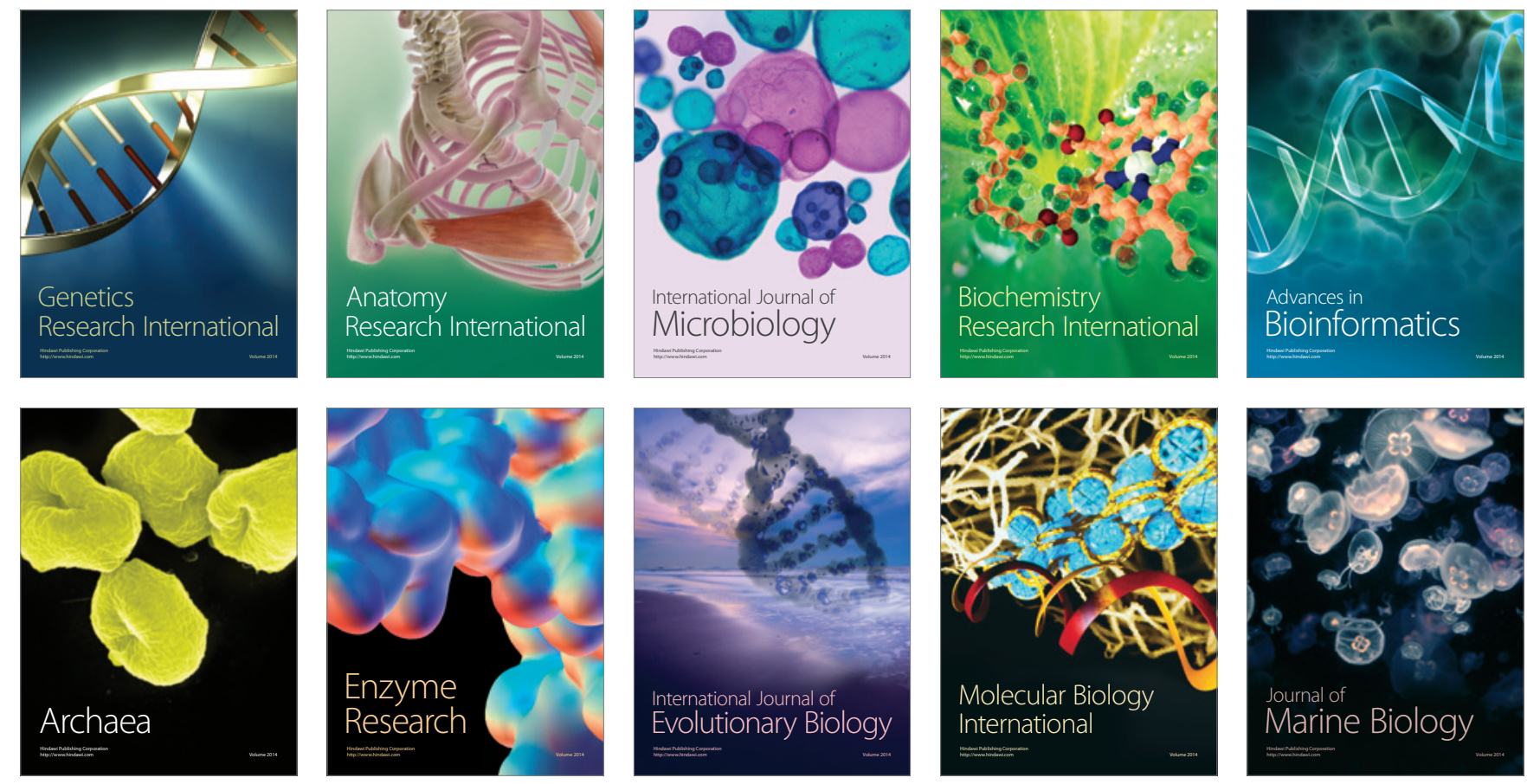